\title{
Fóruns de Discussão em Ambientes Virtuais de Aprendizagem: Um Mapeamento Sistemático do seu Uso nos Contextos Brasileiro e Latino-Americano
}

\author{
Ithalo R. A. Soares ${ }^{1,2}$, Priscilla B. Mendes $^{1}$, Josiane L. Machiavelli ${ }^{1}$, Cristine M. G. \\ de Gusmão ${ }^{1}$
}

${ }^{1}$ Grupo de Pesquisa SABER Tecnologias Educacionais e Sociais - Universidade Federal de Pernambuco (UFPE) - Recife - PE - Brasil

${ }^{2}$ Centro de Informática - Universidade Federal de Pernambuco (UFPE) - Recife - PE Brasil

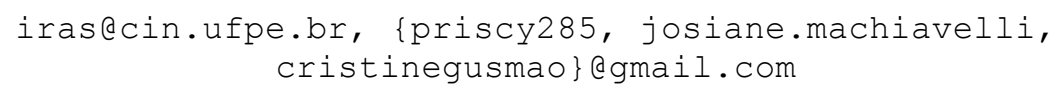

\begin{abstract}
To better understand the use of the forum discussion tool in virtual learning environments in the Brazilian and Latin American contexts, this paper presents results of a systematic mapping conducted from the analysis of full articles from periodicals and book chapters, in Portuguese and Spanish languages, found in databases that aggregate several publications in these languages. The findings suggest that the discussion forum has been used as a resource to facilitate online teaching-learning processes by promoting a space for interaction and collaboration between course participants.
\end{abstract}

Resumo. Com objetivo de melhor compreender a utilização da ferramenta fórum de discussão em ambientes virtuais de aprendizagem nos contextos brasileiro e latino-americano, o presente artigo apresenta resultados de um mapeamento sistemático conduzido a partir da análise de artigos completos de periódicos e capítulos de livros, nos idiomas português e espanhol, encontrados em bases de dados que agregam diversas publicações nesses idiomas. Os achados sugerem que o fórum de discussão tem sido utilizado como um recurso facilitador dos processos de ensino-aprendizagem online por promover um espaço de interação e colaboração entre os participantes dos cursos.

\section{Introdução}

Nos últimos anos, a educação on-line tem adquirido popularidade em vários níveis educacionais, sobretudo nos cursos superiores. O uso das plataformas virtuais de ensino, que para alguns aparenta ser algo novo e inesperado, já vem sendo sinalizado pela literatura há aproximadamente duas décadas [Batista e Gobara 2006], [Oliveira e Filho 2006], [Pepa 2006]. Independentemente do contexto ser presencial, semipresencial ou a distância, percebem-se os ambientes virtuais de aprendizagem como espaços de grande importância nos processos de ensino e aprendizagem mediados por tecnologia. É nesse cenário de plataformas e-learning que várias ferramentas demonstram ser de grande valia na difusão do conhecimento, podendo-se destacar o fórum de discussão, uma ferramenta assíncrona que fomenta a aprendizagem colaborativa. Ao considerar a interseção entre TDICs (Tecnologias Digitais de Informação e Comunicação) e 
educação, com recorte para fóruns de discussão educacional, decidiu-se fazer uma investigação mais específica por intermédio de um mapeamento sistemático da literatura, no intuito de contribuir com pesquisas futuras que envolvam o uso desta ferramenta, procurando esclarecer suas características e indicações de utilização.

O presente artigo dispõe-se em cinco seções. Após esta introdução, fundamentam-se os conceitos de ambientes virtuais de aprendizagem e fóruns de discussão. A seção três especifica os protocolos e técnicas utilizadas na pesquisa. Em seguida, na seção quatro, discutem-se os dados extraídos. A seção cinco encerra este artigo com as considerações finais e perspectivas futuras.

\section{Referencial Teórico}

\subsection{Ambiente Virtual de Aprendizagem}

O ambiente virtual de aprendizagem (AVA), também conhecido como LMS - acrônimo para o termo em inglês Learning Management System, é um sistema educacional idealizado para facilitar os processos que envolvem o ensino on-line. Segundo Almeida (2003), são sistemas destinados ao suporte de atividades mediadas por TDICs, que facilitam a integração de mídias e recursos, permitindo a interação entre pessoas e objetos de conhecimento. Oliveira e Nascimento (2015), por sua vez, salientam que a internet dispõe de outros ambientes que, mesmo sem a complexidade de um AVA, também podem ser utilizados como espaço de aprendizagem virtual. Se por um lado observa-se a adaptação de redes sociais e similares como ambientes educacionais, por outro verifica-se o avanço das pesquisas e melhorias de plataformas existentes.

Estes softwares oferecem um conjunto de recursos que permitem o desenvolvimento de atividades no tempo, espaço e ritmo de cada participante [Ribeiro et al, 2007]. Dispondo de ferramentas síncronas e assíncronas, os ambientes virtuais de aprendizagem possibilitam o uso de diferentes recursos, os quais são combinados no processo de design dos cursos visando atingir os objetivos pedagógicos definidos. Dentre uma variedade de instrumentos, incluindo chats, wikis e questionários, destacase o fórum de discussão, que consiste em uma ferramenta assíncrona cujo objetivo é facilitar a interação e aprendizagem colaborativa.

\subsection{Fóruns de discussão na Educação}

Para Brito (2017) os meios de comunicação têm influenciado nosso modo de viver e estudar, e é nessa perspectiva que o fórum de discussão se apresenta como ferramenta com potencial para otimizar os meios de construção de conhecimento. Acompanhando o avanço histórico, o fórum agora ocupa um espaço digital e é incorporado a websites e ambientes virtuais de aprendizagem, facilitando a troca de ideias e a construção colaborativa do conhecimento. Sánchez (2005) define o fórum virtual como espaço de comunicação no qual as mensagens são classificadas de acordo com sua temática, possibilitando aos alunos fazerem novas contribuições no tempo-espaço que lhe for mais oportuno, pois eles são de natureza assíncrona, o que flexibiliza o aprendizado em diferentes contextos. Apesar de todos os benefícios apontados, Kratochwill e Silva (2008), sinalizam que o fórum de discussão em AVAs propicia os processos de construção do conhecimento sob a perspectiva colaborativa se os objetivos dos docentes e discentes estiverem alinhados a este propósito, ou seja, disciplina e comprometimento mútuo são fatores elementares para que a construção do conhecimento seja eficiente. 
Em suma, pode-se definir o fórum virtual de discussão como um espaço no qual a troca de informações é a base da construção do aprendizado, uma vez que ela transita entre os participantes à medida que a interação é estimulada.

\section{Protocolo de Pesquisa}

Segundo Falbo (2008), mapeamentos sistemáticos visam prover uma visão geral de um tópico de pesquisa mais amplo, além de identificar tanto clusters de estudos que podem ser apropriados para investigações mais específicas, quanto subtópicos em que mais estudos primários são necessários. Utilizando esta metodologia, este artigo objetivou compreender o uso de fóruns de discussão em ambientes virtuais de aprendizagem nos contextos brasileiro e latino-americano. Diante deste cenário, as seguintes questões foram elaboradas para conduzir a investigação: 1. Como os fóruns de discussão são utilizados na perspectiva de seus usuários? 2. Qual o impacto educacional derivado do uso de fóruns de discussão no processo de ensino-aprendizagem?

A estratégia de investigação consistiu em dois momentos complementares, sendo o primeiro uma busca automática e o segundo, manual. Na etapa de planejamento, definiu-se que a pesquisa consistiria em recuperar artigos completos de periódicos e capítulos de livros que compreendessem as áreas de educação e/ou tecnologia, nos idiomas português e/ou espanhol. Não foi imposta restrição a um período de tempo específico, no entanto, entre os resultados selecionados, surgiram publicações a partir do ano de 2006. As bases de dados foram selecionadas de acordo com o número de publicações disponíveis no tema e idiomas abordados, sendo escolhidos o Portal de Periódicos CAPES e as bases Oasisbr e Redalyc. Após a definição da string de busca (conjunto de palavras concatenadas ou não por operadores booleanos, o qual objetiva recuperar publicações que as contenham em seu escopo), a primeira fase da investigação foi baseada na pesquisa automática de publicações utilizando os critérios pré-determinados. A string estabelecida foi forum AND ("ambiente virtual de aprendizagem" OR “ambiente virtual de aprendizaje”).

Ao concluir a leitura completa das publicações que satisfizeram as especificações definidas na primeira fase da investigação, sentiu-se a necessidade de realizar um aprofundamento maior nas pesquisas com a finalidade de assegurar mais resultados relevantes que porventura não tivessem sido encontrados pelos mecanismos de busca automática. A partir deste raciocínio, adicionou-se um processo recursivo de averiguação da referência bibliográfica de cada artigo selecionado no primeiro momento. Foram incluídas novas publicações que apresentassem a palavra "fórum" em seu título e cada novo artigo encontrado foi submetido a todos os processos de revisão que os antecedentes a ele. Repetiu-se esta metodologia até que todas as possibilidades de busca fossem esgotadas.

\section{Extração de dados e discussão de resultados}

Após a execução da primeira estratégia de pesquisa, foram encontradas 117 publicações na plataforma Oasisbr, 238 na Redalyc e 30 no Portal de Periódicos CAPES, das quais se leram títulos e resumos. A seleção inicial retornou 30 publicações que, avaliadas após leitura completa, foram reduzidas para 24. Ao final desse processo, iniciou-se a segunda fase da investigação, a análise das referências bibliográficas das publicações previamente selecionadas, na qual foram encontradas mais 19 publicações que, após 
leitura integral e aplicação dos critérios de exclusão (artigos duplicados ou que não estivessem disponíveis gratuitamente), foram reduzidas a 13. No total, considerando ambas estratégias de busca, 36 publicações foram incluídas na seleção final.

Foram identificadas 25 publicações com aplicações práticas dos fóruns nas áreas de educação e humanidades, 6 na área de saúde, 3 nas ciências exatas e 5 não especificaram o contexto. Notou-se que esse recurso se faz presente em todas as fases educacionais pós ensino médio, como graduação, pós-graduação lato sensu, mestrado e doutorado. Em contrapartida, não foi observada a aplicação prática da ferramenta a alunos da educação básica. Em termos geográficos, o Brasil foi o país com maior número de publicações e casos de uso, totalizando 29 artigos contra apenas 8 originários da Argentina (1), Chile (1), Equador (1), Venezuela (1), Costa Rica (2) e México (2). Observou-se um fluxo de produções maior nos anos de 2006 (4), 2011 (5) e 2014 (6). As Figuras 1 e 2 exibem, respectivamente, dados sobre as plataformas de AVAs utilizadas nos trabalhos selecionados, e as áreas de conhecimento onde foram aplicados, e o Quadro 1 sintetiza os artigos recuperados, explicitando os ambientes utilizados e em quais níveis de ensino.

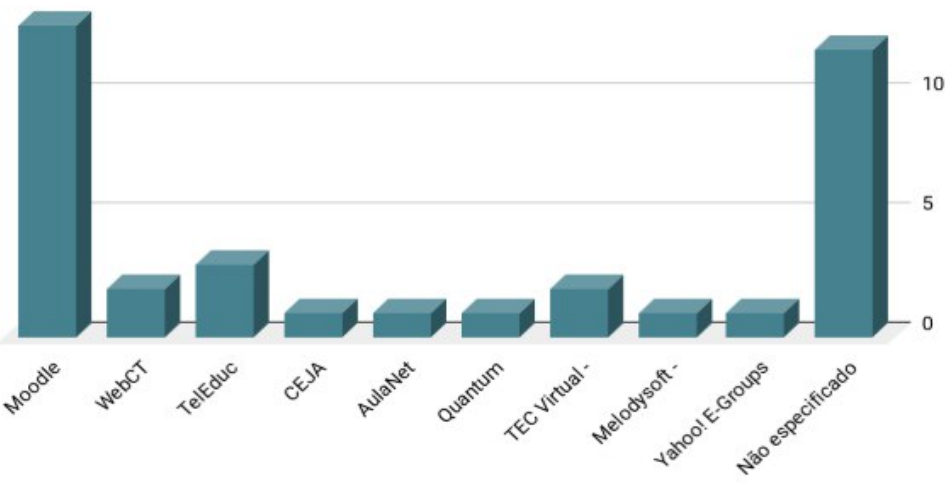

Figura 1. Plataformas de AVAs Fonte: Elaborada pelos autores (2020)

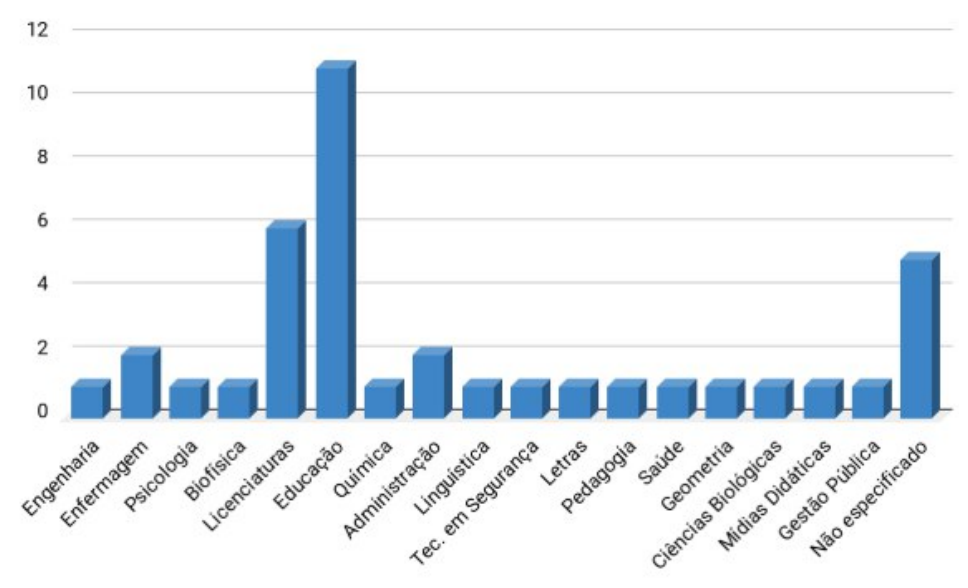

Figura 2. Áreas de conhecimento Fonte: Elaborada pelos autores (2020)

Identificou-se que o Moodle - acrônimo para Modular Object Oriented Dynamic Learning Environment - foi o sistema com mais casos dentre as publicações que especificaram as plataformas utilizadas, sobretudo no Brasil. Disseminado em todo o mundo, o sistema possui uma vasta comunidade de desenvolvedores, que progressivamente realiza melhorias em seu código, que é aberto. É um ambiente robusto e seguro, com interface gráfica simples e personalizável para diversos contextos.

A literatura aponta o uso de fóruns de discussão em diferentes metodologias de ensino, como na Aprendizagem Baseada em Problema [Oliveira e Schluzen 2012], e áreas do conhecimento, como estatística aplicada [Mantovani et al, 2016], bioquímica [Oliveira 2014] e enfermagem [Dias et al., 2011], [Moreno et al., 2013]. Souza e Junior (2012) corroboram com Santos (2009) quando afirmam que o fórum também é um ambiente de socialização e troca de experiências, onde interagem alunos e professores. 
Tabela 1. Síntese de informações dos artigos selecionados

\begin{tabular}{|c|c|c|c|}
\hline Artigo & País & $\begin{array}{l}\text { Ambiente de } \\
\text { aplicação }\end{array}$ & Nível de ensino \\
\hline $\begin{array}{l}\text { Los foros como estrategia de aprendizaje colaborativo los } \\
\text { posgrados virtuales [Cepeda, L.; Palacios, T., 2012] }\end{array}$ & Equador & $\begin{array}{l}\text { Sistema } e \text {-learning } \\
\text { (não especificado) }\end{array}$ & Mestrado \\
\hline $\begin{array}{l}\text { El aprendizaje del lenguaje científico em un foro virtual } \\
\text { [Gonzáles-Álvarez, L. M. de G., 2010] }\end{array}$ & México & $\begin{array}{l}\text { Sistema } e \text {-learning } \\
\text { (não especificado) }\end{array}$ & Graduação \\
\hline $\begin{array}{l}\text { El uso del foro virtual para desarrollar el aprendizaje } \\
\text { autorregulado de los estudiantes universitarios [Castro, N. M. et } \\
\text { al, 2016] }\end{array}$ & México & $\begin{array}{l}\text { Sistema } e \text {-learning } \\
\text { (não especificado) }\end{array}$ & Graduação \\
\hline $\begin{array}{l}\text { Fóruns no ambiente Teleduc: reflexões sobre o papel dos } \\
\text { mediadores e estratégias de gerenciamento de debates [Funo, L. } \\
\text { B. A., Elstermann, A., Souza, M. G., 2015] }\end{array}$ & Brasil & TelEduc & $\begin{array}{l}\text { Curso de formação continuada } \\
\text { para professores de espanhol }\end{array}$ \\
\hline $\begin{array}{l}\text { Ambiente Virtual de Aprendizagem como ferramenta para o } \\
\text { estudo extra-classe e educação continuada [Dias, D. C. et al., } \\
\text { 2011] }\end{array}$ & Brasil & Moodle & $\begin{array}{l}\text { Curso de atualização para } \\
\text { professores, alunos e } \\
\text { profissionais de enfermagem }\end{array}$ \\
\hline $\begin{array}{l}\text { A contribuição de um Ambiente Virtual de Aprendizagem para } \\
\text { potencializar a colaboração no desenvolvimento da } \\
\text { Aprendizagem Baseada em Problemas [Sousa, S. De O. E } \\
\text { Junior, K. S., 2012] }\end{array}$ & Brasil & TelEduc & Graduação \\
\hline $\begin{array}{l}\text { Avaliação da aprendizagem on-line: contribuições específicas } \\
\text { da interface fórum [Kratochwill, S. e SILVA, M., 2008] }\end{array}$ & Brasil & $\begin{array}{l}\text { Plataforma de } \\
\text { aprendizagem on- } \\
\text { line do Consórcio } \\
\text { CEDERJ }\end{array}$ & Graduação \\
\hline $\begin{array}{l}\text { Fórum de discussão como ferramenta no ensino de } \\
\text { administração: um estudo em uma disciplina de estatística } \\
\text { aplicada [Mantovani, D. et al, 2016] }\end{array}$ & Brasil & $\begin{array}{l}\text { Sistema } e \text {-learning } \\
\text { (não especificado) }\end{array}$ & Graduação \\
\hline $\begin{array}{l}\text { Tecnologia na avaliação da aprendizagem colaborativa on-line: } \\
\text { contribuição do fórum de discussão [Segenreichc, S. C. D., } \\
\text { 2008] }\end{array}$ & Brasil & AulaNet & Mestrado \\
\hline $\begin{array}{l}\text { A utilização do fórum de EaD ou fórum online enquanto } \\
\text { instrumento educacional na modalidade de educação a distância } \\
\text { (EaD) [De Brito, D. O., 2017] }\end{array}$ & Brasil & Moodle & Graduação \\
\hline $\begin{array}{l}\text { O ensino-aprendizagem online de Bioquímica e as ferramentas } \\
\text { de mediação: um estudo de caso [Ferreira, A. O. et al., 2014] }\end{array}$ & Brasil & Moodle & Graduação \\
\hline $\begin{array}{l}\text { Fórum EAD e o (des)conhecimento da ferramenta pelos atores } \\
\text { do processo de ensino e aprendizagem [Stelzer, Joana et al, } \\
\text { 2014] }\end{array}$ & Brasil & $\begin{array}{l}\text { Sistema } e \text {-learning } \\
\text { (não especificado) }\end{array}$ & Graduação \\
\hline $\begin{array}{l}\text { Interação nos ambientes virtuais de aprendizagem: análise de } \\
\text { dois fóruns de discussão [Laguardia, J. et al, 2009] }\end{array}$ & Brasil & $\begin{array}{l}\text { Sistema } e \text {-learning } \\
\text { (não especificado) }\end{array}$ & Especialização \\
\hline $\begin{array}{l}\text { Mediação pedagógica em fóruns virtuais: um olhar sobre uma } \\
\text { prática [Castro, E. C. e Freitas, L. G. De, 2013] }\end{array}$ & Brasil & $\begin{array}{l}\text { Sistema } e \text {-learning } \\
\text { (não especificado) }\end{array}$ & Graduação \\
\hline $\begin{array}{l}\text { A produção do material didático e o uso do fórum em AVA } \\
\text { para a formação de professores [Vieira, A. N. G. e Flain, A. L. }\end{array}$ & Brasil & Moodle & Graduação \\
\hline
\end{tabular}




\begin{tabular}{|c|c|c|c|}
\hline G., 2014] & & & \\
\hline $\begin{array}{l}\text { A construção das identidades dos alunos de EaD através dos } \\
\text { seus discursos em um fórum de discussão [Abrão, R. K., Silva, } \\
\text { J. De S. Da, Silva, J. A. Da, 2011] }\end{array}$ & Brasil & Moodle & Pós-graduação lato sensu \\
\hline $\begin{array}{l}\text { O fórum - lugar da interação, da leitura e algo mais [Coelho, I. } \\
\text { Da S., 2014] }\end{array}$ & Brasil & Moodle & Graduação \\
\hline $\begin{array}{l}\text { O fórum livre como recurso de melhoria da relação tutor-aluno } \\
\text { no ambiente virtual: a experiência do 'bote a boca no } \\
\text { trombone...!' [Santos, F. S. Dos, 2009] }\end{array}$ & Brasil & Moodle & Graduação \\
\hline $\begin{array}{l}\text { Dialogismos nos fóruns de discussão na educação on-line } \\
\text { [Marinho, C. L. De O. E Silva, I. M. M, 2014] }\end{array}$ & Brasil & Moodle & $\begin{array}{l}\text { Curso de aperfeiçoamento } \\
\text { direcionado a servidores } \\
\text { públicos do segmento de } \\
\text { Educação }\end{array}$ \\
\hline $\begin{array}{l}\text { Formação didático-pedagógica em saúde: habilidades } \\
\text { cognitivas desenvolvidas pelos pós-graduandos no ambiente } \\
\text { virtual de aprendizagem [Ruiz-Moreno, L. et al., 2013] }\end{array}$ & Brasil & Moodle & Pós-graduação \\
\hline $\begin{array}{l}\text { Perspectiva dos alunos de um curso superior sobre o uso das } \\
\text { ferramentas e-mail, chat e fórum em um ambiente virtual de } \\
\text { aprendizagem [De Lima, L. G. R., Haguenauer, C. J., 2015] }\end{array}$ & Brasil & $\begin{array}{l}\text { Sala Online do } \\
\text { Portal Espaço GD }\end{array}$ & Graduação \\
\hline $\begin{array}{l}\text { O fórum de apresentação no curso de formação de tutores } \\
\text { CEAD/UFU: um panorama do perfil dos candidatos e sua } \\
\text { interatividade a partir do SNAPP [Giemenes, O. M., 2014] }\end{array}$ & Brasil & Moodle & Curso de formação de tutores \\
\hline $\begin{array}{l}\text { Avaliação de um Web Fórum por meio de rubricas [Ferreira, D. } \\
\text { M. e Da Silva, A. C., 2010] }\end{array}$ & Brasil & $\begin{array}{l}\text { Sistema e-learning } \\
\text { (não especificado) }\end{array}$ & Graduação \\
\hline $\begin{array}{l}\text { A análise do fórum de apresentação como gênero discursivo } \\
\text { [Gimenes, O. M., 2019] }\end{array}$ & Brasil & Moodle & $\begin{array}{l}\text { Curso de formação de tutores } \\
\text { EaD }\end{array}$ \\
\hline $\begin{array}{l}\text { O fórum on-line e a interação em um curso a distância [Batista, } \\
\text { E. M. e Gobara, S. T., 2007] }\end{array}$ & Brasil & $\begin{array}{l}\text { Sistema e-learning } \\
\text { (não especificado) }\end{array}$ & Pós-graduação lato sensu \\
\hline $\begin{array}{l}\text { Fóruns de discussão no Moodle: proposta de apresentação } \\
\text { visual das interações [Rodrigues, E. de A. et al, 2016] }\end{array}$ & Brasil & Moodle & Graduação \\
\hline $\begin{array}{l}\text { Siglo XXI, la universidade, el pensamiento crítico y el foro } \\
\text { virtual [Fedorov, A. N., 2006] }\end{array}$ & Costa Rica & TEC Virtual & Graduação \\
\hline $\begin{array}{l}\text { El foro electónico: una herramienta tecnológica para facilitar el } \\
\text { aprendizaje colaboroativo [Brito, R. V., 2004] }\end{array}$ & Venezuela & Melodysoft & Mestrado \\
\hline $\begin{array}{l}\text { Discussões nos ambientes virtuais de aprendizado colaborativo: } \\
\text { a relevância do espaço proporcionado pelo fórum [De Oliveira, } \\
\text { G. P., 2004] }\end{array}$ & Brasil & WebCT & Pós-graduação \\
\hline $\begin{array}{l}\text { Animação de fóruns virtuais de discussão - novo caminho para } \\
\text { a aprendizagem em EaD via web [Oliveira, S. da C. e Filho, G. } \\
\text { J. De L., 2006] }\end{array}$ & Brasil & $\begin{array}{l}\text { Sistema e-learning } \\
\text { (não especificado) }\end{array}$ & Graduação/Pós-graduação \\
\hline $\begin{array}{l}\text { Dialogicidade em fóruns de discussão: interfaces com os } \\
\text { desafios da educação a distância [Marinho, C. L. de O. e } \\
\text { SILVA, I. M. M., 2012] }\end{array}$ & Brasil & Moodle & Pós-graduação lato sensu \\
\hline Interação em fóruns de EaD: a otimização de um espaço de & Brasil & Sistema e-learning & Graduação \\
\hline
\end{tabular}




\begin{tabular}{|l|l|l|l|}
\hline aprendizagem colaborativa [Pereira, S. V. M., 2011] & & (não especificado) & \\
\hline $\begin{array}{l}\text { La utilización de foros virtuales e la universidad como } \\
\text { metodología de aprendizaje colaborativo [Pepa, M. A. T. De, } \\
\text { 2006] }\end{array}$ & Argentina & Yahoo! e-groups & Graduação \\
\hline $\begin{array}{l}\text { El uso del foro de discusión virtual em la enseñanza [Gutiérrez, } \\
\text { D. O., 2007] }\end{array}$ & Mexico & $\begin{array}{l}\text { Sistema e-learning } \\
\text { (não especificado) }\end{array}$ & Não especificado \\
\hline $\begin{array}{l}\text { Foro virtual como una estratégica metodológica para el } \\
\text { desarrollo del pensamiento crítico em la universidad [Fedorov, } \\
\text { A. N., 2006] }\end{array}$ & Costa Rica & TEC virtual & Graduação \\
\hline $\begin{array}{l}\text { O fórum em um ambiente virtual de aprendizado colaborativo } \\
\text { [Oliveira, G. P., 2011] }\end{array}$ & Brasil & WebCT & Pós-graduação \\
\hline
\end{tabular}

A atuação dos docentes se dá no papel de provocar a discussão e estimular o engajamento, e neste sentido, Funo, Elstermann e Souza (2015) sugerem que o potencial para motivar, direcionar e organizar as contribuições dos aprendizes se faz presente desde as postagens de abertura dos fóruns, ficando a cargo dos educadores planejar-se de modo a ampliar ou limitar as possibilidades de participação e interação nesse contexto. A atividade de fórum costuma ser usada como uma proposta de discussão de alguma temática relacionada aos conteúdos dos cursos, mas que pode ser ampliada por estes, em certa medida. Apesar disso, os discentes nem sempre reconhecem a importância deste recurso, atendo-se ao nível de participação mínimo determinado como requisito do curso, conforme relata Mantovani, Gouvêa e Viana (2016).

A atividade de fórum demonstra ser de grande impacto no contexto do ensino on-line, pois estimulou os alunos a refletirem sobre seu próprio pensamento e analisar sua produção textual [Souza; Junior 2012]. A troca de ideias e experiências que pode se dar nesses ambientes geram uma contribuição valiosa ao ensino-aprendizagem, auxiliando na produção do conhecimento coletivo e no fortalecimento de laços sociais, em um espaço em que nem sempre existe um contato mais estreito entre os participantes. No entanto, é necessária a participação ampla dos usuários, docentes e discentes, para que a pluralidade de seus saberes possa se consolidar nesta interface de aprendizagem, conforme sugere Kratochwill e Silva (2008).

\section{Considerações finais}

Constatou-se que a comunidade acadêmica tem direcionado esforços para a pesquisa e desenvolvimento da ferramenta fórum, utilizada como espaço de discussão em ambientes virtuais de aprendizagem, que pode ser aplicada em diversas áreas de conhecimento, como atividade formativa, de avaliação, ou um ambiente de promoção de interações sociais. Para a aprendizagem colaborativa, apresenta benefícios significativos, já que permite que os usuários compartilhem o seu conhecimento sobre o assunto proposto, enriquecendo-o com suas ideias e experiências. Neste sentido, é fundamental que o docente esteja preparado para mediar e estimular os alunos a elevarem o nível dos debates, de modo que a troca de informações facilitada pelas discussões sejam o motto dos processos de ensino-aprendizagem, orientando-os a desenvolver o pensamento crítico e analítico. Assim, desde o primeiro contato com a plataforma, deve estar claro para o aluno que a construção do conhecimento no AVA é 
coletiva, e suas contribuições equivalem em importância às dos professores.

Ao observar que os fóruns de discussão online, e os AVAs, de uma maneira geral, não têm sido utilizados na educação básica, mesmo com todas as benesses apontadas pela literatura em outros níveis de ensino, este artigo busca encorajar educadores a investigarem mais os instrumentos tecnológicos disponíveis na esfera educacional, no intuito de os utilizarem como estímulo para estudantes desta e das próximas gerações, expostos cada vez mais precocemente ao meio digital. Por fim, este estudo objetivou contribuir com pesquisas futuras acerca da ferramenta fórum, estimular novas investigações e fomentar o uso eficaz das ferramentas e plataformas já existentes, uma vez considerada a crescente disseminação da educação online.

\section{Referências}

Abrão, R. K., Da Silva, J. de S., Da Silva, J. A. (2011) “A construção identidades dos alunos de EaD através dos seus discursos em um fórum de discussão", RENOTERevista Novas Tecnologias na Educação, v. 9, n. 1.

Arango, M. (2003) "Foros virtuales como estrategia de aprendizaje", Revista Debates Latinoamericanos, v. 2, n. 7.

Batista, E. e Gobara, S. (2007) "O fórum on-line e a interação em um curso a distância", RENOTE-Revista Novas Tecnologias na Educação, v. 5, n. 1.

Brito, V. (2004) "El foro electrónico: una herramienta tecnológica para facilitar el aprendizaje colaborativo", Edutec. Revista electrónica de tecnología educativa, n. 17, p. a038-a038.

Castro, M. N., Suárez, C. X., Soto, E. V. (2016) "El uso del foro virtual para desarrollar el aprendizaje autorregulado de los estudiantes universitarios", Innovación educativa (México, DF), v. 16, n. 70, p. 23-41.

Castro, E. e De Freitas, L. (2013) "Mediação Pedagógica Em Fóruns Virtuais: Um Olhar Sobre Uma Prática”, XI Congresso Nacional de Educação EDUCERE.

Da Costa, O. S. e Lucena, F. G. J. (2006) "Animação de fóruns virtuais de discussãonovo caminho para a aprendizagem em EAD via web", RENOTE-Revista Novas Tecnologias na Educação, v. 4, n. 2.

Da Silva, C. I. (2014) “O fórum - lugar da interação, da leitura e de algo mais”, Revista Paidéi@-Revista Científica de Educação a Distância, v. 5, n.9.

De Almeida, M. E. B. (2003) "Educação a distância na internet: abordagens e contribuições dos ambientes virtuais de aprendizagem", Educação e pesquisa, v. 29, n. 2 , p. 327-340.

De Almeida, R. E. et al. (2016) "Fóruns de discussão no Moodle: proposta de apresentação visual das interações”, SIED: EnPED-Simpósio Internacional de Educação a Distância e Encontro de Pesquisadores em Educação a Distância.

De Brito, D. O. (2017) “A utilização do fórum de EAD ou fórum on-line enquanto instrumento educacional na modalidade de educação a distância (EAD)", Revista Paidéi@-Revista Científica de Educação a Distância, v. 9, n. 15.

De Guadalupe, G. L. M. (2010) "El aprendizaje del lenguaje científico en un foro 
virtual", Científica, v. 14, n. 1, p. 3-8.

De Lima, L. G. R. e Haguenauer, C. J. (2015) "Perspectiva dos alunos de um curso superior sobre o uso das ferramentas e-mail, chat e fórum em um ambiente virtual de aprendizagem", Revista Ibero-Americana de Estudos em Educação, v. 10, n. 1, p. 8397.

De Oliveira, G. P. (2011) "O fórum em um ambiente virtual de aprendizado colaborativo", São Paulo: Associação Brasileira de Educação a Distância.

De Pepa, M. e Adriana, M. (2006) "La utilización de foros virtuales en la universidad como metodología de aprendizaje colaborativo", Revista cognición, v. 8, p. 59-74.

Dias, D. et al. (2011) "Ambiente virtual de aprendizagem como ferramenta para o estudo extra classe e educação continuada", Cogitare Enfermagem, v. 16, n. 3, p. $565-568$.

Dos Santos, F. S. (2009) "O fórum livre como recurso de melhoria da relação tutoraluno no ambiente virtual: a experiência do 'bote a boca no trombone...!'”, Revista Paidéi@-Revista Científica de Educação a Distância,v. 2, n. 1.

Falbo, R. (2018) “Mapeamento Sistemático". v.7, Disponível em: <https://inf.ufes.br/ falbo/files/MP/TP/Sobre_MS.pdf>. Acesso em: 16 jun 2020.

Fëdorov, A. (2006) “Siglo XXI, la universidad, el pensamiento crítico y el foro virtual”, Revista Iberoamericana de Educación, v. 38, n. 5, p. 1-11.

Fëdorov, A. (2006) "Foro virtual como una estrategia metodológica para el desarrollo del pensamiento crítico en la universidad”, Innovación Educativa, v.6, n. 30, p. 6272 .

Ferreira, A. O., Lima, C. A., Hornink, G. G. (2014) "O ensino-aprendizagem online de Bioquímica e as ferramentas de mediação: um estudo de caso", Revista de Ensino de Bioquímica, v. 12, n. 1, p. 5-23.

Ferreira, D. M. e Da Silva, A. C. (2010) "Avaliação de um Web Fórum por meio de Rubricas”, Revista Meta: Avaliação, v. 2, n. 4, p. 87-127.

Funo, L., Elstermann, A., De Souza, M. (2015) "Fóruns no ambiente Teleduc: reflexões sobre o papel dos mediadores e estratégias de gerenciamento de debates", Revista Brasileira de Linguística Aplicada, v. 15, n. 1, p. 31-59.

Gimenes, O. (2019) "A análise do fórum de apresentação como gênero discursivo", Educação: Teoria e Prática, v. 29, n. 60, p. 237-255.

Gimenes, O. (2014) “O fórum de apresentação no curso de formação de tutores CEAD/UFU: um panorama do perfil dos candidatos e sua interatividade a partir do SNAPP”, RIED. Revista Iberoamericana de Educación a Distancia, v. 17, n. 2, p. 275-302.

Gutiérrez, D. O. (2007) "El uso del Foro de Discusión Virtual em la enseñanza", Revista Iberoamericana de Educación, v. 44, n. 4, p. 4-10.

Kratochwill, S. e Silva, M. (2008) "Avaliação da aprendizagem on-line: contribuições específicas da interface fórum”, Revista Diálogo Educacional, v. 8, n. 24, p. 445-458.

Kitchenham, B. (2004) "Procedures for performing systematic reviews" Keele, UK, 
Keele University, v. 33, n. 2004, p. 1-26.

Laguardia, J. (2009) "Interaction in virtual learning environments: an analysis of two discussion forums", RECIIS, v. 3, n. 2, p. 37-40.

Mantovani, D. M. N., Gouvêa, M. A., Viana, A. B. N. (2016) "Fórum de discussão como ferramenta no ensino de administração: um estudo em uma disciplina de estatística aplicada", Revista de Administração da Universidade Federal de Santa Maria, v. 9, n. 4, p. 681-698.

Marinho, C. L. De O. e Silva, I. M. M. (2014) "Dialogismo nos fóruns de discussão na educação on-line", Signum: Estudos da Linguagem, v. 17, n. 2, p. 253-277.

Marinho, C. L. De O. e Silva, I. M. M. (2012) "Dialogicidade em fóruns de discussão: interfaces com os desafios da educação a distância", $4^{\circ}$ Simpósio Hipertexto e Tecnologia na Educação.

Oliveira, F. C. de M. B. e Nascimento, M. D. R. Do. (2015) “Ambientes Virtuais de Aprendizagem", $2^{\text {a }}$ edição. Editora dad Universidade Estadual do Ceará - EdUECE.

Oliveira, G. P. (2004) "Discussões nos ambientes virtuais de aprendizado colaborativo: a relevância do espaço proporcionado pelo fórum", XII ENDIPE. Conhecimento local e conhecimento universal. Curitiba.

Palacios, T. R. e Cepeda, I. L. (2012) "Los foros como estrategia de aprendizaje colaborativo los posgrados virtuales", Universitas, n. 16, p. 145-169.

Pereira, S. V. M. (2011) "Interação em Fóruns de EAD: a otimização de um espaço de aprendizagem colaborativa", Eutomia, v. 1, n. 07.

Ribeiro, E., Mendonça, G., Mendonça, A. (2007) "A importância dos ambientes virtuais de aprendizagem na busca de novos domínios da EAD”, Anais do $13^{\circ}$ Congresso Internacional de Educação a Distância. Curitiba, Brasil.

Ruiz-Moreno, L., Leite, M., Ajzen, C. (2013) "Formação didático-pedagógica em saúde: habilidades cognitivas desenvolvidas pelos pós-graduandos no ambiente virtual de aprendizagem”, Ciência \& Educação (Bauru), v. 19, n. 1, p. 217-229.

Sánchez, L. P. (2005) "El foro virtual como espacio educativo: propuestas diácticas para su uso", Verista Quaderns Digitals Net, v. 40, p. 1-18.

Segenreich, S. C. D. (2008) "Tecnologia na avaliação da aprendizagem colaborativa online: contribuição do fórum de discussão", Revista Tecnologia e Sociedade, v. 4, n. 6 , p. 153-174.

Sousa, S. e Junior, K. (2012) “A contribuição de um Ambiente Virtual de Aprendizagem para potencializar a colaboração no desenvolvimento da Aprendizagem Baseada em Problemas”, El Hombre y la Máquina, n. 40, p. 44-54.

Stelzer, Joana et al. (2014) "Fórum EAD e o (des)conhecimento da ferramenta pelos atores do processo de ensino e aprendizagem", XIV Colóquio Internacionnal de Gestão Universitária - CIGU.

Vieira, A. e Flain, A. (2014) "A produção de material didático e o uso do fórum em AVA para a formação de professores”, Entretextos, v. 14, n. 1. 